\title{
a-Cuts Of Interval-Valued Fuzzy Matrices With Interval-Valued Fuzzy Rows And Columns
}

\author{
V.Pushpalatha, \\ Asst.Professor, Department of Mathematics, Navarasam Arts and Science College For Women, Arachalur, \\ Erode-638101.
}

\begin{abstract}
Fuzzy Matrix (FM) is a very important topic of Fuzzy algebra. In FM, the elements belong to the unit interval $[0,1]$. When the elements of $F M$ are the subintervals of the unit interval $[0,1]$, then the $F M$ is known as Interval-Valued Fuzzy Matrix [ IVFM ] . In IVFM, the membership values of rows and columns are crisp ie. Rows and columns are certain. But, in many real life situations they are also uncertain. So to model these type of uncertain problems, a new type of Interval-Valued Fuzzy Matrices (IVFMs) are called Interval-Valued Fuzzy Matrices with Interval-Valued Fuzzy Rows and Columns (IVFMFRCs). In this paper, some new elementary operators on $\alpha$-cuts of IVFMFRCs are defined. Using these operators, some important theorems are proved.
\end{abstract}

Keywards: $\alpha$-cut, $\alpha$-cuts of interval valued fuzzy matrix, Fuzzy matrix, Fuzzy rows and columns, Interval valued fuzzy matrix.

\section{Introduction}

Real world decision making problems are very often uncertain or vague in a number of ways. In 1965, Zadeh [9] introduced the concept of fuzzy set theory to meet those problems. In FMs, only the elements are certain. But in many real life situations we observed that rows and columns are uncertain. Fuzzy matrices were introduced by M.G.Thomson [8]. A.K.Shyamal and M.Pal introduced Fuzzy Number Matrices. Two new operators and some properties of fuzzy matrices over the new operators are given in [6]. $\alpha$-cuts of Triangular Fuzzy Numbers and $\alpha$-cuts of Triangular Fuzzy Number Matrices are given in [1]. Pal[3] has defined Fuzzy Matrices with Fuzzy Rows and Fuzzy Columns <FMFRCs>. The elements of FMFRCs are non-negative proper fraction. But, when the elements are the subintervals of the unit interval $[0,1]$, then the FM is known as IVFM. In IVFM, the rows and columns are considered as scripts, but we have seen that they may also be uncertain, ie., rows and columns have same membership values. The concept of IVFMs as a generalization of fuzzy matrix was introduced and developed in 2006 by Shyamal and Pal[5] by extending the max-min operation in fuzzy algebra. In these matrices, rows and columns are also fuzzy numbers, ie., unlike Fuzzy Matrices they are also uncertain. In this paper, some new elementary operators on $\alpha$-cuts of IVFMFRCs are defined. Using these operators, some important theorems are proved.

\section{Preliminaries}

Definition 1.1

Some basic operations on interval-valued fuzzy numbers are given below.

Let $\mathrm{D}$ denote the set of all subintervals of the interval $[0,1]$. Let $\mathrm{a}=\left[\mathrm{a}^{-}, \mathrm{a}^{+}\right]$and $\mathrm{b}=\left[\mathrm{b}^{-}, \mathrm{b}^{+}\right]$be two elements of D. Then

1) $\mathrm{a} \oplus \mathrm{b}=\left[\mathrm{a}^{-}+\mathrm{b}^{-}-\mathrm{a}^{-} \cdot \mathrm{b}^{-}, \mathrm{a}^{+}+\mathrm{b}^{+}-\mathrm{a}^{+} \cdot \mathrm{b}^{+}\right]$,

2) $\mathrm{a} \ominus \mathrm{b}=\left[\mathrm{a}^{-} \ominus \mathrm{b}^{-}, \mathrm{a}^{+} \ominus \mathrm{b}^{+}\right]$, where $\mathrm{a} \ominus \mathrm{b}= \begin{cases}\mathrm{a} & \text { if } \mathrm{a}>\mathrm{b} \\ 0 & \text { if } \mathrm{a} \leq \mathrm{b}\end{cases}$

3) $a \vee b=\left[a^{-}, a^{+}\right] \vee\left[b^{-}, b^{+}\right]=\left[a^{-} \vee b^{-}, a^{+} \vee b^{+}\right]$. where $a \vee b=\max \{x, y\}$

The operators, "+ " and " - " used in extreme right are ordinary addition, subtraction respectively.

Two intervals $\left[\mathrm{a}^{-}, \mathrm{a}^{+}\right]$and $\left[\mathrm{b}^{-}, \mathrm{b}^{+}\right]$are equal if and only ifa- $=\mathrm{b}^{-}$anda ${ }^{+}=\mathrm{b}^{+}$. We denote $[0,0]$ and $[1,1]$ as $\mathbf{0}$ and $\mathbf{1}$ respectively.

\section{Definition 1.2 [5]}

An Interval-Valued Fuzzy Matrix of order $m \times n$ is defined as, $A=\left(a_{i j}\right)_{m \times n}$, where $a_{i j}=\left[a_{i j}^{-}, a_{i j}^{+}\right]$is the ijth element of A, represents the membership value. All the elements of IVFM are intervals and they are members of D.

Definition 1.3 [2]

Let $A=\left[r_{A}(i)\right]\left[c_{A}(j)\right]\left[a_{i j}\right]_{m \times n}$ be an IVFMFRC of order $m \times n$. Here $a_{i j}, i=1,2 \ldots . . m, j=1,2, \ldots n$ represents the $\mathrm{ij}^{\text {th }}$ element of $A, r_{A}(i), c_{A}(j)$ represents the membership values of $i^{\text {th }}$ row and $j^{\text {th }}$ column respectively for $i=$ $1,2 \ldots . \mathrm{m}, \mathrm{j}=1,2, \ldots \mathrm{n}$. $\quad \mathrm{c}_{\mathrm{A}}(1) \mathrm{c}_{\mathrm{A}}(2) \ldots \mathrm{c}_{\mathrm{A}}(\mathrm{m})$ 


$$
\text { Let } \left.\quad A=\begin{array}{c}
r_{A}(2) \\
\vdots \\
r_{A}(n)
\end{array} \begin{array}{cccc}
a_{21} a_{22} & \ldots & a_{2 n} \\
a_{m 1} a_{m 2} & \ldots & a_{m n}
\end{array}\right] \text { be a matrix, where } r_{A}(i), i=1,2, \ldots m, c_{A}(j), j=
$$

$1,2 \ldots \mathrm{n} a_{i j}, \mathrm{i}=1,2, \ldots \mathrm{m}, \mathrm{j}=1,2 \ldots . \mathrm{n}$ represent respectively the membership values of rows, columns and elements.

\section{Definition 1.4}

Let $A=\left[r_{A}(i)\right]\left[c_{A}(j)\right]\left[a_{i j}\right]_{m \times n}$ and $B=\left[r_{B}(i)\right]\left[c_{B}(j)\right]\left[b_{i j}\right]_{m \times n}$ be two IVMFRCs of order $m \times n$. Then the following operators are defined

1) $A \oplus B=\left[\left[r_{A}(i)\right]\left[c_{A}(j)\right]\left[a_{i j}\right]_{m \times n}\right] \oplus\left[\left[r_{B}(i)\right]\left[c_{B}(j)\right]\left[b_{i j}\right]_{m \times n}\right]$

2) $A \vee B=\left[\left[r_{A}(i)\right]\left[c_{A}(j)\right]\left[a_{i j}\right]_{m \times n}\right] \vee\left[\left[r_{B}(i)\right]\left[c_{B}(j)\right]\left[b_{i j}\right]_{m \times n}\right]$

3) $\left.\mathrm{A} \ominus B=\left[\left[\mathrm{r}_{\mathrm{A}}(\mathrm{i})\right]\left[\mathrm{c}_{\mathrm{A}}(\mathrm{j})\right]\left[\mathrm{a}_{\mathrm{ij}}\right]_{\mathrm{m} \times \mathrm{n}}\right] \ominus\left[\mathrm{r}_{\mathrm{B}}(\mathrm{i})\right]\left[\mathrm{c}_{\mathrm{B}}(\mathrm{j})\right]\left[\mathrm{b}_{\mathrm{ij}}\right]_{\mathrm{m} \times \mathrm{n}}\right]$

4) $A \geq B$ iff $\left[r_{A}(i)\right] \geq\left[r_{B}(i)\right], \quad\left[c_{A}(j)\right] \geq\left[c_{B}(j)\right],\left[a_{i j}\right]_{m \times n} \geq\left[b_{i j}\right]_{m \times n}$

\section{Definition 1.5}

The Upper $\boldsymbol{\alpha}$-cut of an IVFMFRC A $=\left[\mathrm{r}_{\mathrm{A}}(\mathrm{i})\right]\left[\mathrm{c}_{\mathrm{A}}(\mathrm{j})\right]\left[\mathrm{a}_{\mathrm{ij}}\right]_{\mathrm{m} \times \mathrm{n}}$ is defined as

$$
\mathrm{A}^{(\alpha)}=\left[\mathrm{r}_{\mathrm{A}}^{(\alpha)}(\mathrm{i})\right]\left[\mathrm{c}_{\mathrm{A}}^{(\alpha)}(\mathrm{j})\right]\left[\mathrm{a}_{\mathrm{ij}}^{(\alpha)}\right]_{\mathrm{m} \times \mathrm{n}}
$$

Here $r_{A}(i)$ and $c_{A}(j)$ represents the membership values of $i^{\text {th }}$ row and $j^{\text {th }}$ column respectively for $i=1,2, \ldots m, j=$ $1,2, \ldots . . n$.

Here, $a_{i j}, i=1,2, \ldots m, j=1,2 \ldots n$ represents the $\mathrm{ij}^{\text {th }}$ elements of $\mathrm{A}$.

$a_{i j}^{(\alpha)}=\left[a_{i j}^{-(\alpha)}, a_{i j}^{+(\alpha)}\right]=[1,1]$ if $a_{i j}^{-(\alpha)}, a_{i j}^{+(\alpha)} \geq \alpha$

$$
\begin{gathered}
{[0,1] \text { if } \mathrm{a}_{i j}^{-(\alpha)}<\alpha \text { and } \mathrm{a}_{\mathrm{ij}}^{+(\alpha)} \geq \alpha} \\
{[0,0] \text { if } \mathrm{a}_{i \mathrm{j}}^{-(\alpha)}, \mathrm{a}_{\mathrm{ij}}^{+(\alpha)}<\alpha} \\
\mathrm{r}_{\mathrm{A}}^{(\alpha)}(\mathrm{i})=\left[\mathrm{r}_{\mathrm{A}}^{-(\alpha)}(\mathrm{i}), \mathrm{r}_{\mathrm{A}}^{+(\alpha)}(\mathrm{i})\right]=[1,1] \text { if } \mathrm{r}_{\mathrm{A}}^{-(\alpha)}(\mathrm{i}), \mathrm{r}_{\mathrm{A}}^{+(\alpha)}(\mathrm{i}) \geq \alpha \\
{[0,1] \text { if } \mathrm{r}_{\mathrm{A}}^{-(\alpha)}(\mathrm{i})<\alpha \text { and } \mathrm{r}_{\mathrm{A}}^{+(\alpha)}(\mathrm{i}) \geq \alpha} \\
{[0,0] \text { f r r }_{\mathrm{A}}^{-(\alpha)}(\mathrm{i}), \mathrm{r}_{\mathrm{A}}^{+(\alpha)}(\mathrm{i})<\alpha} \\
\mathrm{c}_{\mathrm{A}}^{(\alpha)}(\mathrm{j})=\left[\mathrm{c}_{\mathrm{A}}^{-(\alpha)}(\mathrm{j}), \mathrm{c}_{\mathrm{A}}^{+(\alpha)}(\mathrm{j})\right]=[1,1] \text { if } \mathrm{c}_{\mathrm{A}}^{-(\alpha)}(\mathrm{j}), \mathrm{c}_{\mathrm{A}}^{+(\alpha)}(\mathrm{j}) \geq \alpha \\
{[0,1] \text { if } \mathrm{c}_{\mathrm{A}}^{-(\alpha)}(\mathrm{j})<\alpha \text { and c} \mathrm{c}_{\mathrm{A}}^{+(\alpha)}(\mathrm{j}) \geq \alpha} \\
{[0,0] \text { if } \mathrm{c}_{\mathrm{A}}^{-(\alpha)}(\mathrm{j}), \mathrm{c}_{\mathrm{A}}^{+(\alpha)}(\mathrm{j})<\alpha}
\end{gathered}
$$

\section{Definition 1.6}

The Lower $\boldsymbol{\alpha}$-cut of an IVFMFRC

$\mathrm{A}=\left[\mathrm{r}_{\mathrm{A}}(\mathrm{i})\right]\left[\mathrm{c}_{\mathrm{A}}(\mathrm{j})\right]\left[\mathrm{a}_{\mathrm{ij}}\right]_{\mathrm{m} \times \mathrm{n}}$ is defined as

$A_{(\alpha)}=\left[\mathrm{r}_{\mathrm{A}(\alpha)}(\mathrm{i})\right]\left[\mathrm{c}_{\mathrm{A}(\alpha)}(\mathrm{j})\right]\left[\mathrm{a}_{\mathrm{ij}(\alpha)}\right]_{m \times n}$

Here $r_{A}(i)$ and $c_{A}(j)$ represents the membership values of $i^{\text {th }}$ row and $j^{\text {th }}$ column respectively for $i=1,2, \ldots \mathrm{m}, j=$ $1,2, \ldots . . \mathrm{n}$.

Here, $a_{i j}, i=1,2, \ldots m, j=1,2 \ldots n$ represents the $i j^{\text {th }}$ elements of A.

$$
\begin{aligned}
& \mathrm{a}_{\mathrm{ij}(\alpha)}=\left[\mathrm{a}_{\mathrm{ij}-(\alpha)}, \mathrm{a}_{\mathrm{ij}+(\alpha)}\right]=\left[\mathrm{a}_{\mathrm{ij}-(\alpha)}, \mathrm{a}_{\mathrm{ij}+(\alpha)}\right] \text { if } \mathrm{a}_{\mathrm{ij}-(\alpha)}, \mathrm{a}_{\mathrm{ij}+(\alpha)} \geq \alpha \\
& =\left[0, \mathrm{a}_{\mathrm{ij}+(\alpha)}\right] \text { if } \mathrm{a}_{\mathrm{ij}-(\alpha)}<\alpha, \mathrm{a}_{\mathrm{ij}+(\alpha)} \geq \alpha \\
& =[0,0] \text { if } \mathrm{a}_{\mathrm{ij}-(\alpha)}, \mathrm{a}_{\mathrm{ij}+(\alpha)}<\alpha \\
& \mathrm{r}_{\mathrm{A}(\alpha)}(\mathrm{i})=\left[\mathrm{r}_{\mathrm{A}-(\alpha)}(\mathrm{i}), \mathrm{r}_{\mathrm{A}+(\alpha)}(\mathrm{i})\right]=\left[\mathrm{r}_{\mathrm{A}-(\alpha)}(\mathrm{i}), \mathrm{r}_{\mathrm{A}+(\alpha)}(\mathrm{i})\right] \text { if } \mathrm{r}_{\mathrm{A}-(\alpha)}(\mathrm{i}), \mathrm{r}_{\mathrm{A}+(\alpha)}(\mathrm{i}) \geq \alpha \\
& =\left[0, \mathrm{r}_{\mathrm{A}+(\alpha)}(\mathrm{i})\right] \text { if } \mathrm{r}_{\mathrm{A}-(\alpha)}(\mathrm{i})<\alpha, \mathrm{r}_{\mathrm{A}+(\alpha)}(\mathrm{i}) \geq \alpha \\
& =[0,0] \text { if } \mathrm{r}_{\mathrm{A}-(\alpha)}(\mathrm{i}), \mathrm{r}_{\mathrm{A}+(\alpha)}(\mathrm{i})<\alpha \\
& \mathrm{c}_{\mathrm{A}(\alpha)}(\mathrm{j})=\left[\mathrm{c}_{\mathrm{A}-(\alpha)}(\mathrm{j}), \mathrm{c}_{\mathrm{A}+(\alpha)}(\mathrm{j})\right]=\left[\mathrm{c}_{\mathrm{A}-(\alpha)}(\mathrm{j}), \mathrm{c}_{\mathrm{A}+(\alpha)}(\mathrm{j})\right] \text { if } \mathrm{c}_{\mathrm{A}-(\alpha)}(\mathrm{j}), \mathrm{c}_{\mathrm{A}+(\alpha)}(\mathrm{j}) \geq \alpha \\
& =\left[0, \mathrm{c}_{\mathrm{A}+(\alpha)}(\mathrm{j})\right] \text { if } \mathrm{c}_{\mathrm{A}-(\alpha)}(\mathrm{j})<\alpha, \mathrm{c}_{\mathrm{A}+(\alpha)}(\mathrm{j})<\alpha \\
& =[0,0] \text { ifc }_{\mathrm{A}-(\alpha)}(\mathrm{j}), \mathrm{c}_{\mathrm{A}+(\alpha)}(\mathrm{j})<\alpha
\end{aligned}
$$

\section{Example 1.7}

Consider the IVFMFRCs as follows 


$$
\left.\begin{array}{cccc}
{[0.6,0.9]} & {[0.7,1]} & {[.2,0.5]} \\
\mathrm{A}=\quad[0.4,0.8][[0.2,0.7] & {[0.2,0.7]} & {[0.0,0.4]} \\
{[0.2,0.7]} & {[0.1,0.5]} & {[0.1,0.6]} & {[0.0,0.2]} \\
{[0.6,0.7]} & {[0.3,0.6]} & {[0.4,0.5]} & {[0.1,0.3]}
\end{array}\right]
$$

Then by taking $\alpha=0.5$, we get

$\begin{array}{rlll}{[1,1]} & {[1,1]} & {[0,1]} \\ A^{\alpha}=\quad[0,1][[0,1] & {[0,1]} & {[0,0]} \\ {[0,1]} & {[1,1][0,1]} & {[0,1]} & {[0,0]} \\ {[0,1]} & {[0,1]} & {[0,0]}\end{array}$

and

$[0.6,0.9] \quad[0.7,1] \quad[0,0.5]$

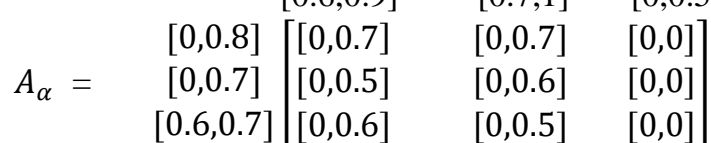

\section{Operator on IVFMFRCs}

\section{Definition 2.1}

Let $A=\left[r_{A}(i)\right]\left[c_{A}(j)\right]\left[a_{i j}\right]_{m \times n}$ and $B=\left[r_{B}(i)\right]\left[c_{B}(j)\right]\left[b_{i j}\right]_{m \times n}$ be two IVMFRCs, then, $A \vee B$ is defined as $A \vee B=D=\left[r_{D}(i)\right]\left[c_{D}(j)\right]\left[d_{i j}\right]_{m \times n}$

where, $r_{D}(i)=r_{A}(i) \vee r_{B}(i)=\left[r_{A}^{-}(i) \vee r_{B}^{-}(i), r_{A}^{+}(i) \vee r_{B}^{+}(i)\right]$

$$
c_{D}(j)=c_{A}(j) \vee c_{B}(j)=\left[c_{A}^{-}(j) \vee c_{B}^{-}(j), c_{A}^{+}(j) \vee c_{B}^{+}(j)\right]
$$

Theorem 2.2

and $d_{i j}=a_{i j} \vee b_{i j}=\left[a_{i j}^{-} \vee b_{i j}^{-}, a_{i j}^{+} \vee b_{i j}^{+}\right]$for all $i, j$.

Proof:

$$
\text { If } A \text { and } B \text { are two IVFMFRCs, then }(A \vee B)^{(\alpha)}=A^{(\alpha)} \vee B^{(\alpha)}
$$

Let $A=\left[r_{A}(i)\right]\left[c_{A}(j)\right]\left[a_{i j}\right]_{m \times n}$ and $B=\left[r_{B}(i)\right]\left[c_{B}(j)\right]\left[b_{i j}\right]_{m \times n}$ be two IVMFRCs, then $A \vee B=D=\left[r_{D}(i)\right]\left[c_{D}(j)\right]\left[d_{i j}\right]_{m \times n}$

where, $r_{D}(i)=r_{A}(i) \vee r_{B}(i)=\left[r_{A}^{-}(i) \vee r_{B}^{-}(i), r_{A}^{+}(i) \vee r_{B}^{+}(i)\right]$

$c_{D}(j)=c_{A}(j) \vee c_{B}(j)=\left[c_{A}^{-}(j) \vee c_{B}^{-}(j), c_{A}^{+}(j) \vee c_{B}^{+}(j)\right]$

and $d_{i j}=a_{i j} \vee b_{i j}=\left[a_{i j}^{-} \vee b_{i j}^{-}, a_{i j}^{+} \vee b_{i j}^{+}\right]$for all $i, j$.

Here the order of $\mathrm{A}$ and $\mathrm{B}$ must be equal.

Let $\mathrm{E}_{\mathrm{ij}}$ and $\mathrm{F}_{\mathrm{ij}}$ be the $\mathrm{ij}^{\text {th }}$ element of $(\mathrm{A} \vee \mathrm{B})^{(\alpha)}$ and $\mathrm{A}^{(\alpha)} \vee \mathrm{B}^{(\alpha)}$

Therefore, $E_{i j}=(A \vee B)^{(\alpha)}$ and $F_{i j}=A^{(\alpha)} \vee B^{(\alpha)}$

Case 1:

$$
\mathrm{A} \geq \mathrm{B} \geq \alpha
$$

ie., $\left[r_{A}(i)\right]\left[c_{A}(j)\right]\left[a_{i j}\right]_{m \times n} \geq\left[r_{B}(i)\right]\left[c_{B}(j)\right]\left[b_{i j}\right]_{m \times n} \geq a$

$\Rightarrow\left[\mathrm{r}_{\mathrm{A}}(\mathrm{i})\right] \geq\left[\mathrm{r}_{\mathrm{B}}(\mathrm{i})\right] \geq \alpha,\left[\mathrm{c}_{\mathrm{A}}(\mathrm{j})\right] \geq\left[\mathrm{c}_{\mathrm{B}}(\mathrm{j})\right] \geq \alpha, \mathrm{a}_{\mathrm{ij}} \geq \mathrm{b}_{\mathrm{ij}} \geq \alpha$

$\mathrm{E}_{\mathrm{ij}}=(\mathrm{A} \vee \mathrm{B})^{(\alpha)}$

$=\left(\left[\mathrm{r}_{\mathrm{D}}(\mathrm{i})\right]\left[\mathrm{c}_{\mathrm{D}}(\mathrm{j})\right]\left[\mathrm{d}_{\mathrm{ij}}\right]_{\mathrm{m} \times \mathrm{n}}\right)^{(\alpha)}$

$=\left(\left[\mathrm{r}_{\mathrm{A}}(\mathrm{i})\right]\left[\mathrm{c}_{\mathrm{A}}(\mathrm{j})\right]\left[\mathrm{a}_{\mathrm{ij}}\right]_{\mathrm{m} \times \mathrm{n}}\right)^{(\alpha)}$

$=\left[\mathrm{r}_{\mathrm{A}}^{(\alpha)}(\mathrm{i})\right]\left[\mathrm{c}_{\mathrm{A}}^{(\alpha)}(\mathrm{j})\right]\left[\mathrm{a}_{\mathrm{ij}}^{(\alpha)}\right]_{m \times n}$

$\left.=\left[\mathrm{r}_{\mathrm{A}}^{-(\alpha)}(\mathrm{i}), \mathrm{r}_{\mathrm{A}}^{+(\alpha)}(\mathrm{i})\right] \mathrm{c}_{\mathrm{A}}^{-(\alpha)}(\mathrm{j}), \mathrm{c}_{\mathrm{A}}^{+(\alpha)}(\mathrm{j})\right]\left[\mathrm{a}_{\mathrm{ij}}^{-(\alpha)}, \mathrm{a}_{\mathrm{ij}}^{+(\alpha)}\right]$

$=[1,1][1,1][1,1]_{\mathrm{m} \times \mathrm{n}}$

$F_{i j}=A^{(\alpha)} \vee B^{(\alpha)}$

$=\left(\left[\mathrm{r}_{\mathrm{A}}(\mathrm{i})\right]\left[\mathrm{c}_{\mathrm{A}}(\mathrm{j})\right]\left[\mathrm{a}_{\mathrm{ij}}\right]_{\mathrm{m} \times \mathrm{n}}\right)^{(\alpha)} \vee\left(\left[\mathrm{r}_{\mathrm{B}}(\mathrm{i})\right]\left[\mathrm{c}_{\mathrm{B}}(\mathrm{j})\right]\left[\mathrm{b}_{\mathrm{ij}}\right]_{\mathrm{m} \times \mathrm{n}}\right)^{(\alpha)}$

$=\left(\left[\mathrm{r}_{\mathrm{A}}^{(\alpha)}(\mathrm{i})\right]\left[\mathrm{c}_{\mathrm{A}}^{(\alpha)}(\mathrm{j})\right]\left[a_{i j}^{(\alpha)}\right]_{m \times n}\right) \vee\left(\left[\mathrm{r}_{\mathrm{B}}^{(\alpha)}(\mathrm{i})\right]\left[\mathrm{c}_{\mathrm{B}}^{(\alpha)}(\mathrm{j})\right]\left[\mathrm{b}_{i j}^{(\alpha)}\right]_{m \times n}\right)$

$=\left[\mathrm{r}_{\mathrm{A}}^{(\alpha)}(\mathrm{i}) \vee \mathrm{r}_{\mathrm{B}}^{(\alpha)}(\mathrm{i})\right]\left[\mathrm{c}_{\mathrm{A}}^{(\alpha)}(\mathrm{j}) \vee \mathrm{c}_{\mathrm{B}}^{(\alpha)}(\mathrm{j})\right]\left[a_{i j}^{(\alpha)} \vee \mathrm{b}_{i j}^{(\alpha)}\right]_{m \times n}$

$=[1,1][1,1][1,1]_{\mathrm{m} \times \mathrm{n}}$

From (2.2.1) and (2.2.2), $(\mathrm{A} \vee \mathrm{B})^{(\alpha)}=\mathrm{A}^{(\alpha)} \vee \mathrm{B}^{(\alpha)}$

Case 2:

$\mathrm{A} \geq \alpha \geq \mathrm{B}$

$\left[\mathrm{r}_{\mathrm{A}}(\mathrm{i})\right]\left[\mathrm{c}_{\mathrm{A}}(\mathrm{j})\right]\left[\mathrm{a}_{\mathrm{ij}}\right]_{\mathrm{m} \times \mathrm{n}} \geq \alpha \geq\left[\mathrm{r}_{\mathrm{B}}(\mathrm{i})\right]\left[\mathrm{c}_{\mathrm{B}}(\mathrm{j})\right]\left[\mathrm{b}_{\mathrm{ij}}\right]_{\mathrm{m} \times \mathrm{n}}$ 
ie., $r_{A}(i) \geq \alpha \geq r_{B}(i), c_{A}(j) \geq \alpha \geq c_{B}(j), a_{i j} \geq \alpha \geq b_{i j}$

Let $E_{i j}=(A \vee B)^{(\alpha)}$

$$
\begin{aligned}
& =\left(\left[\mathrm{r}_{\mathrm{D}}(\mathrm{i})\right]\left[\mathrm{c}_{\mathrm{D}}(\mathrm{j})\right]\left[\mathrm{d}_{\mathrm{ij}}\right]_{\mathrm{m} \times \mathrm{n}}\right)^{(\alpha)} \\
& =\left(\left[\mathrm{r}_{\mathrm{A}}(\mathrm{i})\right]\left[\mathrm{c}_{\mathrm{A}}(\mathrm{j})\right]\left[\mathrm{a}_{\mathrm{ij}}\right]_{\mathrm{m} \times \mathrm{n}}\right)^{(\alpha)} \\
& =\left[\mathrm{r}_{\mathrm{A}}^{(\alpha)}(\mathrm{i})\right]\left[\mathrm{c}_{\mathrm{A}}^{(\alpha)}(\mathrm{j})\right]\left[\mathrm{a}_{\mathrm{ij}}^{(\alpha)}\right]_{\mathrm{m} \times \mathrm{n}} \\
& =\left[\mathrm{r}_{\mathrm{A}}^{(\alpha)}(\mathrm{i}) \vee \mathrm{r}_{\mathrm{B}}^{(\alpha)}(\mathrm{i})\right]\left[\mathrm{c}_{\mathrm{A}}^{(\alpha)}(\mathrm{j}) \vee \mathrm{c}_{\mathrm{B}}^{(\alpha)}(\mathrm{j})\right]\left[a_{i j}^{(\alpha)} \vee \mathrm{b}_{i j}^{(\alpha)}\right]_{m \times n} \\
& =[1,1][1,1][1,1]_{\mathrm{m} \times \mathrm{n}} \\
& \mathrm{F}_{\mathrm{ij}}=\mathrm{A}^{(\alpha)} \vee \mathrm{B}^{(\alpha)} \\
& =\left(\left[\mathrm{r}_{\mathrm{A}}(\mathrm{i})\right]\left[\mathrm{c}_{\mathrm{A}}(\mathrm{j})\right]\left[\mathrm{a}_{\mathrm{ij}}\right]_{\mathrm{m} \times \mathrm{n}}\right)^{(\alpha)} \vee\left(\left[\mathrm{r}_{\mathrm{B}}(\mathrm{i})\right]\left[\mathrm{c}_{\mathrm{B}}(\mathrm{j})\right]\left[\mathrm{b}_{\mathrm{ij}}\right]_{\mathrm{m} \times \mathrm{n}}\right)^{(\alpha)} \\
& =\left[\mathrm{r}_{\mathrm{A}}^{(\alpha)}(\mathrm{i})\right]\left[\mathrm{c}_{\mathrm{A}}^{(\alpha)}(\mathrm{j})\right]\left[a_{i j}^{(\alpha)}\right]_{m \times n} \vee\left[\mathrm{r}_{\mathrm{B}}^{(\alpha)}(\mathrm{i})\right]\left[\mathrm{c}_{\mathrm{B}}^{(\alpha)}(\mathrm{j})\right]\left[\mathrm{b}_{i j}^{(\alpha)}\right]_{m \times n} \\
& =\left[\mathrm{r}_{\mathrm{A}}^{(\alpha)}(\mathrm{i}) \vee \mathrm{r}_{\mathrm{B}}^{(\alpha)}(\mathrm{i})\right]\left[\mathrm{c}_{\mathrm{A}}^{(\alpha)}(\mathrm{j}) \vee \mathrm{c}_{\mathrm{B}}^{(\alpha)}(\mathrm{j})\right]\left[a_{i j}^{(\alpha)} \vee \mathrm{b}_{i j}^{(\alpha)}\right]_{m \times n} \\
& =[1 \vee 0,1 \vee 0][1 \vee 0,1 \vee 0][1 \vee 0,1 \vee 0] \\
& =[1,1][1,1][1,1]
\end{aligned}
$$

From (2.2.3) and (2.2.4), $(A \vee B)^{(\alpha)}=A^{(\alpha)} \vee B^{(\alpha)}$

Case 3 :

$$
\begin{aligned}
& \alpha>\mathrm{A}>\mathrm{B} \\
& \alpha>\left[r_{A}(i)\right]\left[c_{A}(j)\right]\left[a_{i j}\right]_{m \times n} \geq\left[r_{B}(i)\right]\left[c_{B}(j)\right]\left[b_{i j}\right]_{m \times n} \\
& \mathrm{E}_{\mathrm{ij}}=(\mathrm{A} \vee \mathrm{B})^{(\alpha)} \\
& =\left(\left[r_{D}(\mathrm{i})\right]\left[c_{D}(j)\right]\left[d_{i j}\right]_{m \times n}\right)^{(\alpha)} \\
& =\left(\left[\mathrm{r}_{\mathrm{A}}(\mathrm{i})\right]\left[\mathrm{c}_{\mathrm{A}}(\mathrm{j})\right]\left[\mathrm{a}_{\mathrm{ij}}\right]_{\mathrm{m} \times \mathrm{n}}\right)^{(\alpha)} \\
& =\left[\mathrm{r}_{\mathrm{A}}^{(\alpha)}(\mathrm{i})\right]\left[\mathrm{c}_{\mathrm{A}}^{(\alpha)}(\mathrm{j})\right]\left[a_{i j}^{(\alpha)}\right]_{m \times n} \\
& =\left[\mathrm{r}_{\mathrm{A}}^{(\alpha)}(\mathrm{i}) \vee \mathrm{r}_{\mathrm{B}}^{(\alpha)}(\mathrm{i})\right]\left[\mathrm{c}_{\mathrm{A}}^{(\alpha)}(\mathrm{j}) \vee \mathrm{c}_{\mathrm{B}}^{(\alpha)}(\mathrm{j})\right]\left[a_{i j}^{(\alpha)} \vee \mathrm{b}_{i j}^{(\alpha)}\right]_{m \times n} \\
& =[0,0][0,0][0,0]_{m \times n} \\
& \mathrm{~F}_{\mathrm{ij}}=\mathrm{A}^{(\alpha)} \vee \mathrm{B}^{(\alpha)} \\
& =\left[\mathrm{r}_{\mathrm{A}}^{(\alpha)}(\mathrm{i}) \vee \mathrm{r}_{\mathrm{B}}^{(\alpha)}(\mathrm{i})\right]\left[\mathrm{c}_{\mathrm{A}}^{(\alpha)}(\mathrm{j}) \vee \mathrm{c}_{\mathrm{B}}^{(\alpha)}(\mathrm{j})\right]\left[a_{i j}^{(\alpha)} \vee \mathrm{b}_{i j}^{(\alpha)}\right]_{m \times n} \\
& =[0 \vee 0,0 \vee 0][0 \vee 0,0 \vee 0][0 \vee 0,0 \vee 0] \\
& =[0,0][0,0][0,0]
\end{aligned}
$$

From (2.2.5) and (2.2.6), $(\mathrm{A} \vee \mathrm{B})^{(\alpha)}=\mathrm{A}^{(\alpha)} \vee \mathrm{B}^{(\alpha)}$

In all three cases, $(A \vee B)^{(\alpha)}=A^{(\alpha)} \vee B^{(\alpha)}$

\section{Definition 2.3}

Let Let $A=\left[r_{A}(i)\right]\left[c_{A}(j)\right]\left[a_{i j}\right]_{m \times n}$ and $B=\left[r_{B}(i)\right]\left[c_{B}(j)\right]\left[b_{i j}\right]_{m \times n}$ be two IVMFRCs, then $\mathbf{A} \oplus \mathbf{B}$ is defined as $A \oplus B=D=\left[r_{D}(i)\right]\left[c_{D}(j)\right]\left[d_{i j}\right]_{m \times n}$

$$
\text { where, } \begin{aligned}
r_{D}(i)= & r_{A}(i) \bigoplus r_{B}(i)=\left[r_{A}^{-}(i)+r_{B}^{-}(i)-r_{A}^{-}(i) r_{B}^{-}(i), r_{A}^{+}(i)+r_{B}^{+}(i)-r_{A}^{+}(i) r_{B}^{+}(i)\right] \\
& c_{D}(j)=c_{A}(j) \oplus c_{B}(j)=\left[c_{A}^{-}(j)+c_{B}^{-}(j)-c_{A}^{-}(j) c_{B}^{-}(j), c_{A}^{+}(j)+c_{B}^{+}(j)-c_{A}^{+}(j) c_{B}^{+}(j)\right] \\
& d_{i j}=a_{i j} \oplus b_{i j}=\left[a_{i j}^{-}+b_{i j}^{-}-a_{i j}^{-} b_{i j}^{-}, a_{i j}^{+}+b_{i j}^{+}-a_{i j}^{+} b_{i j}^{+}\right]
\end{aligned}
$$

\section{Theorem 2.4}

\section{Proof :}

$$
\text { If } \mathrm{A} \text { and } \mathrm{B} \text { are two IVFMFRCs, then }(\mathrm{A} \oplus \mathrm{B})^{(\alpha)} \geq \mathrm{A}^{(\alpha)} \oplus \mathrm{B}^{(\alpha)}
$$

\section{Case 1:}

Let $\mathrm{G}_{\mathrm{ij}}$ and $\mathrm{H}_{\mathrm{ij}}$ be the $(\mathrm{ij})^{\text {th }}$ element of $(\mathrm{A} \oplus \mathrm{B})^{(\alpha)}$ and $A^{(\alpha)} \oplus \mathrm{B}^{(\alpha)}$.

ie., $\left[r_{A}(i)\right]\left[c_{A}(j)\right]\left[a_{i j}\right]_{m \times n} \geq\left[r_{B}(i)\right]\left[c_{B}(j)\right]\left[b_{i j}\right]_{m \times n} \geq \alpha$

$\mathrm{G}_{\mathrm{ij}}=(\mathrm{A} \oplus \mathrm{B})^{(\alpha)}$

$$
\begin{aligned}
& =\left(\left[\mathrm{r}_{\mathrm{D}}(\mathrm{i})\right]\left[\mathrm{c}_{\mathrm{D}}(\mathrm{j})\right]\left[\mathrm{d}_{\mathrm{ij}}\right]_{\mathrm{m} \times \mathrm{n}}\right)^{(\alpha)} \\
& =\left(\left[\mathrm{r}_{\mathrm{A}}(\mathrm{i}) \oplus \mathrm{r}_{\mathrm{B}}(\mathrm{i})\right]\left[\mathrm{c}_{\mathrm{A}}^{-}(\mathrm{j}) \oplus \mathrm{c}_{\mathrm{A}}^{+}(\mathrm{j})\right]\left[\mathrm{a}_{\mathrm{ij}}^{-} \oplus \mathrm{a}_{\mathrm{ij}}^{+}\right]_{m \times n}\right)^{(\alpha)}
\end{aligned}
$$

$=\left(\left[r_{A}^{-}(i)+r_{B}^{-}(i)-r_{A}^{-}(i) r_{B}^{-}(i), r_{A}^{+}(i)+r_{B}^{+}(i)-r_{A}^{+}(i) r_{B}^{+}(i)\right]\right.$

$\left.\left.c_{A}^{-}(j) c_{B}^{-}(j), c_{A}^{+}(j)+c_{B}^{+}(j)-c_{A}^{+}(j) c_{B}^{+}(j)\right]\left[a_{i j}^{-}+b_{i j}^{-}-a_{i j}^{-} b_{i j}^{-}, a_{i j}^{+}+b_{i j}^{+}-a_{i j}^{+} b_{i j}^{+}\right]\right)^{(\alpha)}$ 
$=\left(\left[r_{A}^{-}(i)+r_{B}^{-}(i)\left(1-r_{A}^{-}(i)\right), r_{A}^{+}(i)+r_{B}^{+}(i)\left(1-r_{A}^{+}(i)\right)\right]\left[c_{A}^{-}(j)+c_{B}^{-}(j)\left(1-c_{A}^{-}(j)\right), c_{A}^{+}(j)+c_{B}^{+}(j)\left(1-c_{A}^{+}(j)\right)\right]\left[a_{i j}^{-}+b_{i j}^{-}\right.\right.$ $\left.\left.\left(1-\mathrm{b}_{\mathrm{ij}}^{-}\right), \mathrm{a}_{\mathrm{ij}}^{+}+\mathrm{b}_{\mathrm{ij}}^{+}\left(1-\mathrm{a}_{\mathrm{ij}}^{+}\right)\right]\right)^{(\alpha)}$

$\geq\left(\left[r_{A}^{-}(\mathrm{i}), r_{A}^{+}(\mathrm{i})\right]\left[c_{A}^{-}(j), c_{A}^{+}(j)\right]\left[a_{i j}^{-}, a_{i j}^{+}\right]\right)(\alpha)$

$>\left(\left[\mathrm{r}_{\mathrm{A}}^{-}(\mathrm{i}), \mathrm{r}_{\mathrm{A}}^{+}(\mathrm{i})\right]^{(\alpha)}\left[\mathrm{c}_{\mathrm{A}}^{-}(\mathrm{j}), \mathrm{c}_{\mathrm{A}}^{+}(\mathrm{j})\right]^{(\alpha)}\left[\mathrm{a}_{\mathrm{ij}}^{-}, \mathrm{a}_{\mathrm{ij}}^{+}\right]_{\mathrm{m} \times \mathrm{n}}^{(\alpha)}\right)$

$=[1,1][1,1][1,1]_{\mathrm{m} \times \mathrm{n}}$

And $\mathrm{H}_{\mathrm{ij}}=A^{(\alpha)} \oplus \mathrm{B}^{(\alpha)}$

$$
\begin{aligned}
= & \left(\left[\mathrm{r}_{\mathrm{A}}(\mathrm{i})\right]\left[\mathrm{c}_{\mathrm{A}}(\mathrm{j})\right]\left[\mathrm{a}_{\mathrm{ij}}\right]_{\mathrm{m} \times \mathrm{n}}\right)^{(\alpha)} \oplus\left(\left[\mathrm{r}_{\mathrm{B}}(\mathrm{i})\right]\left[\mathrm{c}_{\mathrm{B}}(\mathrm{j})\right]\left[\mathrm{b}_{\mathrm{ij}}\right]_{\mathrm{m} \times \mathrm{n}}\right)^{(\alpha)} \\
= & {\left[\mathrm{r}_{\mathrm{A}}^{(\alpha)}(\mathrm{i}) \oplus \mathrm{r}_{\mathrm{B}}^{(\alpha)}(\mathrm{i})\right]\left[\mathrm{c}_{\mathrm{A}}^{(\alpha)}(\mathrm{i}) \oplus \mathrm{c}_{\mathrm{B}}^{(\alpha)}(\mathrm{i})\right]\left[\mathrm{a}_{\mathrm{ij}}^{(\alpha)} \oplus \mathrm{b}_{\mathrm{ij}}^{(\alpha)}\right]_{\mathrm{m} \times \mathrm{n}} } \\
= & {\left[\mathrm{r}_{\mathrm{A}}^{-(\alpha)}(\mathrm{i})+\mathrm{r}_{\mathrm{B}}^{-(\alpha)}(\mathrm{i})-\mathrm{r}_{\mathrm{A}}^{-(\alpha)}(\mathrm{i}) \mathrm{r}_{\mathrm{B}}^{-(\alpha)}(\mathrm{i}), \mathrm{r}_{\mathrm{A}}^{+(\alpha)}(\mathrm{i})+\mathrm{r}_{\mathrm{B}}^{+(\alpha)}(\mathrm{i})-\mathrm{r}_{\mathrm{A}}^{+(\alpha)}(\mathrm{i}) \mathrm{r}_{\mathrm{B}}^{+(\alpha)}(\mathrm{i})\right] } \\
& \quad\left[\mathrm{c}_{\mathrm{A}}^{-(\alpha)}(\mathrm{j})+\mathrm{c}_{\mathrm{B}}^{-(\alpha)}(\mathrm{j})-\mathrm{c}_{\mathrm{A}}^{-(\alpha)}(\mathrm{j}) \mathrm{c}_{\mathrm{B}}^{-(\alpha)}(\mathrm{j}), \mathrm{c}_{\mathrm{A}}^{+(\alpha)}(\mathrm{j})+\mathrm{c}_{\mathrm{B}}^{+(\alpha)}(\mathrm{j})-\mathrm{c}_{\mathrm{A}}^{+(\alpha)}(\mathrm{j}) \mathrm{c}_{\mathrm{B}}^{+(\alpha)}(\mathrm{j})\right] \\
& \quad\left[\mathrm{a}_{\mathrm{ij}}^{-}+\mathrm{b}_{\mathrm{ij}}^{-}-\mathrm{a}_{\mathrm{ij}}^{-} \mathrm{b}_{\mathrm{ij}}^{-}, \mathrm{a}_{\mathrm{ij}}^{+}+\mathrm{b}_{\mathrm{ij}}^{+}-\mathrm{a}_{\mathrm{ij}}^{+} \mathrm{b}_{\mathrm{ij}}^{+}\right] \mathrm{m} \times \mathrm{n} \\
= & {[1+1-1,1+1-1][1+1-1,1+1-1][1+1-1,1+1-1]_{\mathrm{m} \times \mathrm{n}} } \\
= & {[1,1][1,1][1,1]_{\mathrm{m} \times \mathrm{n}} }
\end{aligned}
$$

From (2.4.1) and (2.4.2), $\quad \mathrm{H}_{\mathrm{ij}}>\mathrm{G}_{\mathrm{ij}}$

\section{Case 2:}

$(\mathrm{A} \oplus \mathrm{B})^{(\alpha)}>A^{(\alpha)} \oplus \mathrm{B}^{(\alpha)}$

$$
\mathrm{A} \geq \alpha>\mathrm{B}
$$

ie., $\left[r_{A}(i)\right]\left[c_{A}(j)\right]\left[a_{i j}\right]_{m \times n} \geq \alpha>\left[r_{B}(i)\right]\left[c_{B}(j)\right]\left[b_{i j}\right]_{m \times n}$

$\mathrm{G}_{\mathrm{ij}}=(\mathrm{A} \oplus \mathrm{B})^{(\alpha)}$

$=\left(\left[\mathrm{r}_{\mathrm{D}}(\mathrm{i})\right]\left[\mathrm{c}_{\mathrm{D}}(\mathrm{j})\right]\left[\mathrm{d}_{\mathrm{ij}}\right]_{\mathrm{m} \times \mathrm{n}}\right)^{(\alpha)}$

$=\left(\left[r_{A}^{-}(i) \oplus r_{A}^{+}(i)\right]\left[c_{A}^{-}(j) \oplus c_{A}^{+}(j)\right]\left[a_{i j}^{-}, a_{i j}^{+}\right]_{m \times n}\right)^{(\alpha)}$

$=\left(\left[r_{A}^{-}(i)+r_{B}^{-}(i)-r_{A}^{-}(i) r_{B}^{-}(i), r_{A}^{+}(i)+r_{B}^{+}(i)-r_{A}^{+}(i) r_{B}^{+}(i)\right]\right.$

$\left.\left[c_{A}^{-}(j)+c_{B}^{-}(j)-c_{A}^{-}(j) c_{B}^{-}(j), c_{A}^{+}(j)+c_{B}^{+}(j)-c_{A}^{+}(j) c_{B}^{+}(j)\right]\left[a_{i j}^{-}+b_{i j}^{-}-a_{i j}^{-} b_{i j}^{-}, a_{i j}^{+}+b_{i j}^{+}-a_{i j}^{+} b_{i j}^{+}\right]\right)^{(\alpha)}$

$=\left(\left[r_{A}^{-}(i)+r_{B}^{-}(i)\left(1-r_{A}^{-}(i)\right), r_{A}^{+}(i)+r_{B}^{+}(i)\left(1-r_{A}^{+}(i)\right)\right]\left[c_{A}^{-}(j)+c_{B}^{-}(j)\left(1-c_{A}^{-}(j)\right), c_{A}^{+}(j)+c_{B}^{+}(j)\left(1-\quad c_{A}^{+}(j)\right)\right]\left[a_{i j}^{-}\right.\right.$

$\left.\left.+b_{i j}^{-}\left(1-b_{i j}^{-}\right), a_{i j}^{+}+b_{i j}^{+}\left(1-a_{i j}^{+}\right)\right]\right)^{(\alpha)}$

$\geq\left(\left[r_{A}^{-}(\mathrm{i}), r_{A}^{+}(\mathrm{i})\right]\left[c_{A}^{-}(j), c_{A}^{+}(j)\right]\left[a_{i j}^{-}, a_{i j}^{+}\right]\right)^{(\alpha)}$

$>\left(\left[\mathrm{r}_{\mathrm{A}}^{-}(\mathrm{i}), \mathrm{r}_{\mathrm{A}}^{+}(\mathrm{i})\right]^{(\alpha)}\left[\mathrm{c}_{\mathrm{A}}^{-}(\mathrm{j}), \mathrm{c}_{\mathrm{A}}^{+}(\mathrm{j})\right]^{(\alpha)}\left[\mathrm{a}_{\mathrm{ij}}^{-}, \mathrm{a}_{\mathrm{ij}}^{+}\right]_{\mathrm{m} \times \mathrm{n}}^{(\alpha)}\right.$

$$
=[1,1][1,1][1,1]_{m \times n}
$$

And $\mathrm{H}_{\mathrm{ij}}=A^{(\alpha)} \oplus \mathrm{B}^{(\alpha)}$

$$
\begin{aligned}
= & \left(\left[\mathrm{r}_{\mathrm{A}}(\mathrm{i})\right]\left[\mathrm{c}_{\mathrm{A}}(\mathrm{j})\right]\left[\mathrm{a}_{\mathrm{ij}}\right]_{\mathrm{m} \times \mathrm{n}}\right)^{(\alpha)} \oplus\left(\left[\mathrm{r}_{\mathrm{B}}(\mathrm{i})\right]\left[\mathrm{c}_{\mathrm{B}}(\mathrm{j})\right]\left[\mathrm{b}_{\mathrm{ij}}\right]_{\mathrm{m} \times \mathrm{n}}\right)^{(\alpha)} \\
= & {\left[\mathrm{r}_{\mathrm{A}}^{(\alpha)}(\mathrm{i}) \oplus \mathrm{r}_{\mathrm{B}}^{(\alpha)}(\mathrm{i})\right]\left[\mathrm{c}_{\mathrm{A}}^{(\alpha)}(\mathrm{i}) \oplus \mathrm{c}_{\mathrm{B}}^{(\alpha)}(\mathrm{i})\right]\left[\mathrm{a}_{\mathrm{ij}}^{(\alpha)} \oplus \mathrm{b}_{\mathrm{ij}}^{(\alpha)}\right]_{\mathrm{m} \times \mathrm{n}} } \\
= & {\left[\mathrm{r}_{\mathrm{A}}^{-(\alpha)}(\mathrm{i})+\mathrm{r}_{\mathrm{B}}^{-(\alpha)}(\mathrm{i})-\mathrm{r}_{\mathrm{A}}^{-(\alpha)}(\mathrm{i}) \mathrm{r}_{\mathrm{B}}^{-(\alpha)}(\mathrm{i}), \mathrm{r}_{\mathrm{A}}^{+(\alpha)}(\mathrm{i})+\mathrm{r}_{\mathrm{B}}^{+(\alpha)}(\mathrm{i})-\mathrm{r}_{\mathrm{A}}^{+(\alpha)}(\mathrm{i}) \mathrm{r}_{\mathrm{B}}^{+(\alpha)}(\mathrm{i})\right] } \\
& \quad\left[\mathrm{c}_{\mathrm{A}}^{-(\alpha)}(\mathrm{j})+\mathrm{c}_{\mathrm{B}}^{-(\alpha)}(\mathrm{j})-\mathrm{c}_{\mathrm{A}}^{-(\alpha)}(\mathrm{j}) \mathrm{c}_{\mathrm{B}}^{-(\alpha)}(\mathrm{j}), \mathrm{c}_{\mathrm{A}}^{+(\alpha)}(\mathrm{j})+\mathrm{c}_{\mathrm{B}}^{+(\alpha)}(\mathrm{j})-\mathrm{c}_{\mathrm{A}}^{+(\alpha)}(\mathrm{j}) \mathrm{c}_{\mathrm{B}}^{+(\alpha)}(\mathrm{j})\right] \\
& \quad\left[\mathrm{a}_{\mathrm{ij}}^{-}+\mathrm{b}_{\mathrm{ij}}^{-}-\mathrm{a}_{\mathrm{ij}}^{-} \mathrm{b}_{\mathrm{ij}}^{-}, \mathrm{a}_{\mathrm{ij}}^{+}+\mathrm{b}_{\mathrm{ij}}^{+}-\mathrm{a}_{\mathrm{ij}}^{+} \mathrm{b}_{\mathrm{ij}}^{+}\right]_{\mathrm{m} \times \mathrm{n}}
\end{aligned}
$$

$=[1+0-0,1+0-0][1+0-0,1+0-0][1+0-0,1+0-0]_{m \times n}$

$=[1,1][1,1][1,1]_{\mathrm{m} \times \mathrm{n}}(2.4 .4)$

From (2.4.3) and (2.4.4), $\mathrm{G}_{\mathrm{ij}}>H_{\mathrm{ij}}$

\section{Case 3:}

$(\mathrm{A} \oplus \mathrm{B})^{(\alpha)}>A^{(\alpha)} \oplus \mathrm{B}^{(\alpha)}$

ie., $\alpha>\left[r_{A}(i)\right]\left[c_{A}(j)\right]\left[a_{i j}\right]_{m \times n}>\left[r_{B}(i)\right]\left[c_{B}(j)\right]\left[b_{i j}\right]_{m \times n}$

$\mathrm{G}_{\mathrm{ij}}=(\mathrm{A} \oplus \mathrm{B})^{(\alpha)}$

$=\left(\left[\mathrm{r}_{\mathrm{D}}(\mathrm{i})\right]\left[\mathrm{c}_{\mathrm{D}}(\mathrm{j})\right]\left[\mathrm{d}_{\mathrm{ij}}\right]_{\mathrm{m} \times \mathrm{n}}\right)^{(\alpha)}$

$=\left(\left[r_{A}^{-}(i) \oplus r_{A}^{+}(i)\right]\left[c_{A}^{-}(j) \oplus c_{A}^{+}(j)\right]\left[a_{i j}^{-}, a_{i j}^{+}\right]_{m \times n}\right)^{(\alpha)}$

$=\left(\left[r_{A}^{-}(i)+r_{B}^{-}(i)-r_{A}^{-}(i) r_{B}^{-}(i), r_{A}^{+}(i)+r_{B}^{+}(i)-r_{A}^{+}(i) r_{B}^{+}(i)\right]\right.$

$\left.\left[c_{A}^{-}(j)+c_{B}^{-}(j)-c_{A}^{-}(j) c_{B}^{-}(j), c_{A}^{+}(j)+c_{B}^{+}(j)-c_{A}^{+}(j) c_{B}^{+}(j)\right]\left[a_{i j}^{-}+b_{i j}^{-}-a_{i j}^{-} b_{i j}^{-}, a_{i j}^{+}+b_{i j}^{+}-a_{i j}^{+} b_{i j}^{+}\right]\right)^{(\alpha)}$

$=\left(\left[r_{A}^{-}(i)+r_{B}^{-}(i)\left(1-r_{A}^{-}(i)\right), r_{A}^{+}(i)+r_{B}^{+}(i)\left(1-r_{A}^{+}(i)\right)\right] \quad\left[\quad c_{A}^{-}(j)+c_{B}^{-}(j)\left(1-c_{A}^{-}(j)\right), c_{A}^{+}(j)+c_{B}^{+}(j)\left(1-c_{A}^{+}(j)\right)\right][\right.$ $\left.\left.a_{i j}^{-}+b_{i j}^{-}\left(1-b_{i j}^{-}\right), a_{i j}^{+}+b_{i j}^{+}\left(1-a_{i j}^{+}\right)\right]\right)^{(\alpha)}$

$\geq\left(\left[r_{A}^{-}(\mathrm{i}), r_{A}^{+}(\mathrm{i})\right]\left[c_{A}^{-}(j), c_{A}^{+}(j)\right]\left[a_{i j}^{-}, a_{i j}^{+}\right]\right)^{(\alpha)}$ 


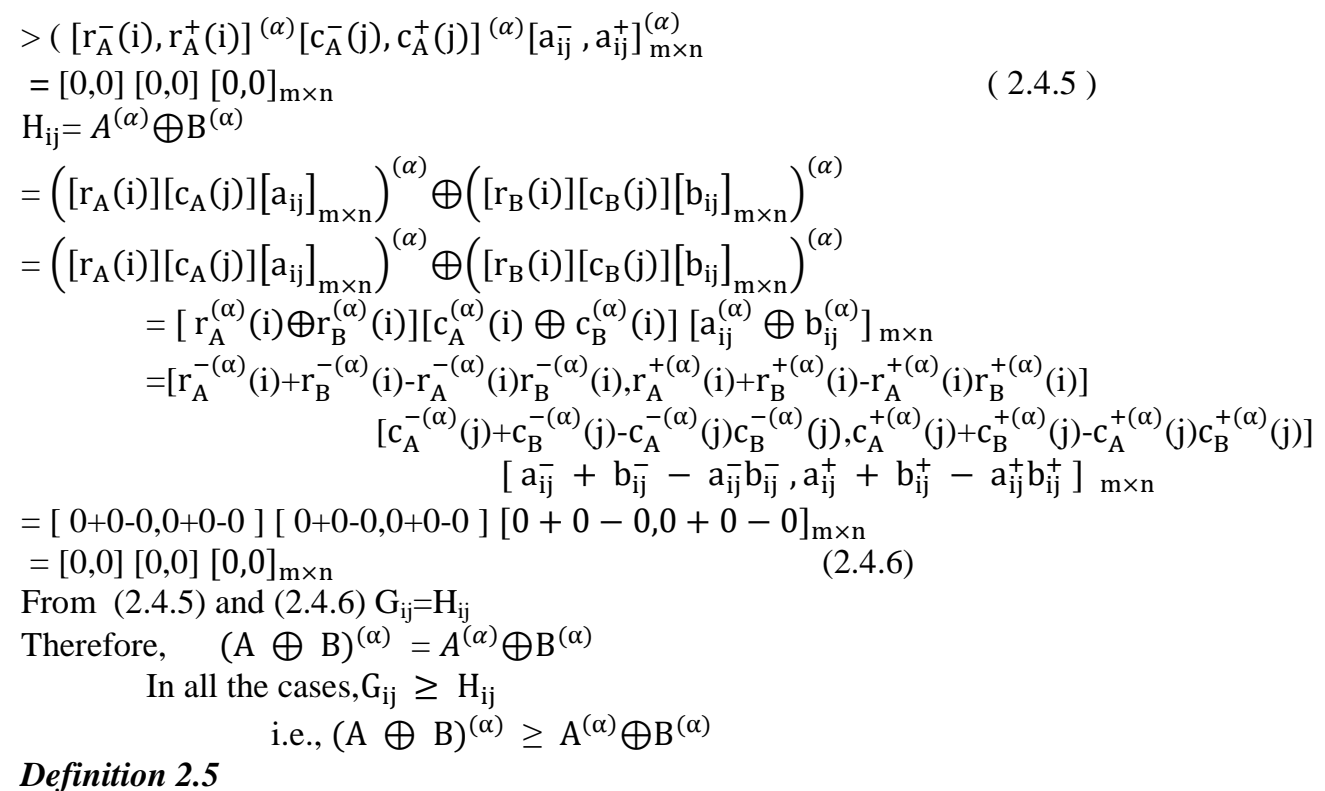

Let $\mathrm{A}=\left[\mathrm{r}_{\mathrm{A}}(\mathrm{i})\right]\left[\mathrm{c}_{\mathrm{A}}(\mathrm{j})\right]\left[\mathrm{a}_{\mathrm{ij}}\right]_{\mathrm{m} \times \mathrm{n}}$ and $\mathrm{B}=\left[\mathrm{r}_{\mathrm{B}}(\mathrm{i})\right]\left[\mathrm{c}_{\mathrm{B}}(\mathrm{j})\right]\left[\mathrm{b}_{\mathrm{ij}}\right]_{\mathrm{m} \times \mathrm{n}}$ be two IVMFRCs, then $\mathrm{A} \ominus \mathrm{B}$ is defined as $A \ominus B=D=\left[r_{D}(i)\right]\left[c_{D}(j)\right]\left[d_{i j}\right]_{m \times n}$

where, $r_{D}(i)=\left[r_{A}(i) \ominus r_{B}(i)\right]=\left[r_{A}^{-}(i) \ominus r_{B}^{-}(i), r_{A}^{+}(i) \Theta r_{B}^{+}(i)\right]$

$c_{D}(j)=\left[c_{A}(j) \Theta c_{B}(j)\right]=\left[c_{A}^{-}(j) \Theta c_{B}^{-}(j), c_{A}^{+}(i) \Theta c_{B}^{+}(j)\right]$

$\mathrm{a}_{\mathrm{ij}}=\left[\mathrm{a}_{\mathrm{ij}} \Theta \mathrm{b}_{\mathrm{ij}}\right]=\left[\mathrm{a}_{\mathrm{ij}}^{-} \Theta \mathrm{b}_{\mathrm{ij}}^{-}, a_{i j}^{+} \ominus \mathrm{b}_{\mathrm{ij}}^{+}\right]$

where, $\quad a_{i j} \ominus b_{i j}=\left[\begin{array}{cc}1 & \text { if } \\ a_{i j} & >b_{i j} \\ 0 & a_{i j} \leq b_{i j}\end{array}\right]$

\section{Theorem 2.6}

If $\mathrm{A}$ and $\mathrm{B}$ are two IVFMFRCs, then $(\mathrm{A} \ominus \mathrm{B})^{(\alpha)} \geq \mathrm{A}^{(\alpha)} \ominus \mathrm{B}^{(\alpha)}$.

\section{Proof:}

Let $\mathrm{A}=\left[\mathrm{r}_{\mathrm{A}}(\mathrm{i})\right]\left[\mathrm{c}_{\mathrm{A}}(\mathrm{j})\right]\left[\mathrm{a}_{\mathrm{ij}}\right]_{\mathrm{m} \times \mathrm{n}}$ and $\mathrm{B}=\left[\mathrm{r}_{\mathrm{B}}(\mathrm{i})\right]\left[\mathrm{c}_{\mathrm{B}}(\mathrm{j})\right]\left[\mathrm{b}_{\mathrm{ij}}\right]_{\mathrm{m} \times \mathrm{n}}$ be two IVMFRCs, then $\mathrm{A} \ominus \mathrm{B}$ is defined as $A \ominus B=D=\left[r_{D}(i)\right]\left[c_{D}(j)\right]\left[d_{i j}\right]_{m \times n}$

where, $r_{D}(i)=\left[r_{A}(i) \Theta r_{B}(i)\right]=\left[r_{A}^{-}(i) \Theta r_{B}^{-}(i), r_{A}^{+}(i) \Theta r_{B}^{+}(i)\right]$

$c_{D}(j)=\left[c_{A}(j) \Theta c_{B}(j)\right]=\left[c_{A}^{-}(j) \Theta c_{B}^{-}(j), c_{A}^{+}(i) \Theta c_{B}^{+}(j)\right]$

$a_{i j}=\left[a_{i j} \ominus b_{i j}\right]=\left[a_{i j}^{-} \ominus b_{i j}^{-}, a_{i j}^{+} \ominus b_{i j}^{+}\right]$

$$
\text { where, } \quad a_{i j} \ominus b_{i j}=\left[\begin{array}{cc}
1 & \text { if } a_{i j}>b_{i j} \\
0 & a_{i j} \leq b_{i j}
\end{array}\right]
$$

Let $\mathrm{K}_{\mathrm{ij}}$ and $\mathrm{L}_{\mathrm{ij}}$ be the $\mathrm{ij}^{\text {th }}$ element of $\mathrm{A}^{(\alpha)} \ominus \mathrm{B}^{(\alpha)}$ and $(\mathrm{A} \ominus \mathrm{B})^{(\alpha)}$

Here, $L_{i j}=(A \ominus B)^{(\alpha)}$ and $K_{i j}=A^{(\alpha)} \ominus B^{(\alpha)}$

Case 1:

$$
\mathrm{A} \geq \mathrm{B} \geq \alpha
$$

ie., $\left[r_{A}(i)\right]\left[c_{A}(j)\right]\left[a_{i j}\right]_{m \times n} \geq\left[r_{B}(i)\right]\left[c_{B}(j)\right]\left[b_{i j}\right]_{m \times n} \geq \alpha$

$$
\begin{aligned}
\mathrm{L}_{\mathrm{ij}} & =(\mathrm{A} \ominus \mathrm{B})^{(\alpha)} \\
& =\left(\left[\mathrm{r}_{\mathrm{D}}(\mathrm{i})\right]\left[\mathrm{c}_{\mathrm{D}}(\mathrm{j})\right]\left[\mathrm{d}_{\mathrm{ij}}\right]_{\mathrm{m} \times \mathrm{n}}\right)^{(\alpha)} \\
& =\left(\left[\mathrm{r}_{\mathrm{A}}(\mathrm{i}) \ominus \mathrm{r}_{\mathrm{B}}(\mathrm{i})\right]\left[\mathrm{c}_{\mathrm{A}}^{-}(\mathrm{j}) \ominus \mathrm{c}_{\mathrm{A}}^{+}(\mathrm{j})\right]\left[\mathrm{a}_{\mathrm{ij}}^{-} \ominus \mathrm{a}_{\mathrm{ij}}^{+}\right]_{m \times n}\right)^{(\alpha)} \\
& =\left(\left[\mathrm{r}_{\mathrm{A}}(\mathrm{i})\right]\left[\mathrm{c}_{\mathrm{A}}(\mathrm{j})\right]\left[\mathrm{a}_{\mathrm{ij}}\right]_{\mathrm{m} \times \mathrm{n}}\right)^{(\alpha)} \\
& =\left[\mathrm{r}_{\mathrm{A}}^{(\alpha)}(\mathrm{i})\right]\left[\mathrm{c}_{\mathrm{A}}^{(\alpha)}(\mathrm{j})\right]\left[\mathrm{a}_{\mathrm{ij}}^{(\alpha)}\right]_{\mathrm{m} \times \mathrm{n}} \\
& \left.=\left[\mathrm{r}_{\mathrm{A}}^{-(\alpha)}(\mathrm{i}), \mathrm{r}_{\mathrm{A}}^{+(\alpha)}(\mathrm{i})\right] \mathrm{c}_{\mathrm{A}}^{-(\alpha)}(\mathrm{j}), \mathrm{c}_{\mathrm{A}}^{+(\alpha)}(\mathrm{j})\right]\left[\mathrm{a}_{\mathrm{ij}}^{(\alpha)}, \mathrm{a}_{\mathrm{ij}}^{+(\alpha)}\right] \\
& =[1,1][1,1][1,1]_{\mathrm{m} \times \mathrm{n}} \\
& \mathrm{K}_{\mathrm{ij}}=\mathrm{A}^{(\alpha)} \ominus \mathrm{B}^{(\alpha)} \\
= & \left(\left[\mathrm{r}_{\mathrm{A}}(\mathrm{i})\right]\left[\mathrm{c}_{\mathrm{A}}(\mathrm{j})\right]\left[\mathrm{a}_{\mathrm{ij}}\right]_{\mathrm{m \times n}}\right)^{(\alpha)} \ominus\left(\left[\mathrm{r}_{\mathrm{B}}(\mathrm{i})\right]\left[\mathrm{c}_{\mathrm{B}}(\mathrm{j})\right]\left[\mathrm{b}_{\mathrm{ij}}\right]_{\mathrm{m} \times \mathrm{n}}\right)^{(\alpha)} \\
= & {\left[\mathrm{r}_{\mathrm{A}}^{(\alpha)}(\mathrm{i}) \ominus \mathrm{r}_{\mathrm{B}}^{(\alpha)}(\mathrm{i})\right]\left[\mathrm{c}_{\mathrm{A}}^{(\alpha)}(\mathrm{i}) \ominus \mathrm{c}_{\mathrm{B}}^{(\alpha)}(\mathrm{i})\right]\left[\mathrm{a}_{\mathrm{ij}}^{(\alpha)} \ominus \mathrm{b}_{\mathrm{ij}}^{(\alpha)}\right]_{\mathrm{m} \times \mathrm{n}} } \\
= & {[1 \ominus 1,1 \ominus 1][1 \ominus 1,1 \ominus 1][1 \ominus 1,1 \ominus 1] }
\end{aligned}
$$


$=[0,0][0,0][0,0]$

From (2.6.1) and (2.6.2) $\mathrm{L}_{\mathrm{ij}}>\mathrm{K}_{\mathrm{ij}}$

Case 2:

$$
(\mathrm{A} \ominus \mathrm{B})^{(\alpha)}>\mathrm{A}^{(\alpha)} \ominus \mathrm{B}^{(\alpha)}
$$

$$
\begin{aligned}
& \mathrm{A} \geq \alpha \geq \mathrm{B} \\
& {\left[\mathrm{r}_{\mathrm{A}}(\mathrm{i})\right]\left[\mathrm{c}_{\mathrm{A}}(\mathrm{j})\right]\left[\mathrm{a}_{\mathrm{ij}}\right]_{\mathrm{m} \times \mathrm{n}} \geq \alpha \geq\left[\mathrm{r}_{\mathrm{B}}(\mathrm{i})\right]\left[\mathrm{c}_{\mathrm{B}}(\mathrm{j})\right]\left[\mathrm{b}_{\mathrm{ij}}\right]_{\mathrm{m} \times \mathrm{n}}} \\
& \text { ie., } r_{A}(i) \geq \alpha \geq r_{B}(i), c_{A}(j) \geq \alpha \geq c_{B}(j), a_{i j} \geq \alpha \geq b_{i j} \\
& \mathrm{~L}_{\mathrm{ij}}=(\mathrm{A} \ominus \mathrm{B})^{(\alpha)} \\
& =\left(\left[\mathrm{r}_{\mathrm{D}}(\mathrm{i})\right]\left[\mathrm{c}_{\mathrm{D}}(\mathrm{j})\right]\left[\mathrm{d}_{\mathrm{ij}}\right]_{\mathrm{m} \times \mathrm{n}}\right)^{(\alpha)} \\
& =\left(\left[\mathrm{r}_{\mathrm{A}}(\mathrm{i}) \ominus \mathrm{r}_{\mathrm{B}}(\mathrm{i})\right]\left[\mathrm{c}_{\mathrm{A}}^{-}(\mathrm{j}) \ominus \mathrm{c}_{\mathrm{A}}^{+}(\mathrm{j})\right]\left[\mathrm{a}_{\mathrm{ij}}^{-} \ominus \mathrm{a}_{\mathrm{ij}}^{+}\right]_{m \times n}\right)^{(\alpha)} \\
& =\left(\left[r_{A}(i)\right]\left[c_{A}(j)\right]\left[a_{i j}\right]_{m \times n}\right)^{(\alpha)} \\
& =\left[\mathrm{r}_{\mathrm{A}}^{(\alpha)}(\mathrm{i})\right]\left[\mathrm{c}_{\mathrm{A}}^{(\alpha)}(\mathrm{j})\right]\left[\mathrm{a}_{\mathrm{ij}}^{(\alpha)}\right]_{\mathrm{m} \times \mathrm{n}} \\
& \left.=\left[r_{A}^{-(\alpha)}(i), r_{A}^{+(\alpha)}(i)\right] c_{A}^{-(\alpha)}(j), c_{A}^{+(\alpha)}(j)\right]\left[a_{i j}^{-(\alpha)}, a_{i j}^{+(\alpha)}\right] \\
& =[1,1][1,1][1,1]_{\mathrm{m} \times \mathrm{n}} \\
& \mathrm{K}_{\mathrm{ij}}=\mathrm{A}^{(\alpha)} \ominus \mathrm{B}^{(\alpha)} \\
& =\left(\left[r_{A}(i)\right]\left[c_{A}(j)\right]\left[a_{i j}\right]_{m \times n}\right)^{(\alpha)} \ominus\left(\left[r_{B}(i)\right]\left[c_{B}(j)\right]\left[b_{i j}\right]_{m \times n}\right)^{(\alpha)} \\
& =\left[r_{\mathrm{A}}^{(\alpha)}(\mathrm{i}) \ominus \mathrm{r}_{\mathrm{B}}^{(\alpha)}(\mathrm{i})\right]\left[\mathrm{c}_{\mathrm{A}}^{(\alpha)}(\mathrm{i}) \ominus \mathrm{c}_{\mathrm{B}}^{(\alpha)}(\mathrm{i})\right]\left[\mathrm{a}_{\mathrm{ij}}^{(\alpha)} \ominus \mathrm{b}_{\mathrm{ij}}^{(\alpha)}\right]_{\mathrm{m} \times \mathrm{n}} \\
& =[1 \ominus 0,1 \ominus 0][1 \ominus 0,1 \ominus 0][1 \ominus 0,1 \ominus 0] \\
& =[1,1][1,1][1,1]
\end{aligned}
$$

From (2.6.3) and (2.6.4),we get

$(\mathrm{A} \ominus \mathrm{B})^{(\alpha)}=\mathrm{A}^{(\alpha)} \ominus \mathrm{B}^{(\alpha)}$.

$$
\mathrm{L}_{\mathrm{ij}}=\mathrm{K}_{\mathrm{ij}}
$$

Case 3:

$$
\begin{aligned}
& \alpha \geq A \geq B \\
& \alpha \geq\left[r_{A}(i)\right]\left[c_{A}(j)\right]\left[a_{i j}\right]_{m \times n} \geq\left[r_{B}(i)\right]\left[c_{B}(j)\right]\left[b_{i j}\right]_{m \times n}
\end{aligned}
$$

ie., $\alpha \geq r_{A}(i) \geq r_{B}(i), \alpha \geq c_{A}(j) \geq c_{B}(j), \alpha \geq a_{i j} \geq b_{i j}$

$\mathrm{L}_{\mathrm{ij}}=(\mathrm{A} \ominus \mathrm{B})^{(\alpha)}$

$$
=\left(\left[\mathrm{r}_{\mathrm{D}}(\mathrm{i})\right]\left[\mathrm{c}_{\mathrm{D}}(\mathrm{j})\right]\left[\mathrm{d}_{\mathrm{ij}}\right]_{\mathrm{m} \times \mathrm{n}}\right)^{(\alpha)}
$$

$=\left(\left[\mathrm{r}_{\mathrm{A}}(\mathrm{i}) \ominus \mathrm{r}_{\mathrm{B}}(\mathrm{i})\right]\left[\mathrm{c}_{\mathrm{A}}^{-}(\mathrm{j}) \ominus \mathrm{c}_{\mathrm{A}}^{+}(\mathrm{j})\right]\left[\mathrm{a}_{\mathrm{ij}}^{-} \ominus \mathrm{a}_{\mathrm{ij}}^{+}\right]_{m \times n}\right)^{(\alpha)}$

$=\left(\left[\mathrm{r}_{\mathrm{A}}(\mathrm{i})\right]\left[\mathrm{c}_{\mathrm{A}}(\mathrm{j})\right]\left[\mathrm{a}_{\mathrm{ij}}\right]_{\mathrm{m} \times \mathrm{n}}\right)^{(\alpha)}$

$$
\begin{aligned}
& =\left[\mathrm{r}_{\mathrm{A}}^{(\alpha)}(\mathrm{i})\right]\left[\mathrm{c}_{\mathrm{A}}^{(\alpha)}(\mathrm{j})\right]\left[\mathrm{a}_{\mathrm{ij}}^{(\alpha)}\right]_{\mathrm{m} \times \mathrm{n}} \\
& \left.=\left[\mathrm{r}_{\mathrm{A}}^{-(\alpha)}(\mathrm{i}), \mathrm{r}_{\mathrm{A}}^{+(\alpha)}(\mathrm{i})\right] \mathrm{c}_{\mathrm{A}}^{-(\alpha)}(\mathrm{j}), \mathrm{c}_{\mathrm{A}}^{+(\alpha)}(\mathrm{j})\right]\left[\mathrm{a}_{\mathrm{ij}}^{-(\alpha)}, \mathrm{a}_{\mathrm{ij}}^{+(\alpha)}\right] \\
& =[0,0][0,0][0,0]
\end{aligned}
$$

$\mathrm{K}_{\mathrm{ij}}=\mathrm{A}^{(\alpha)} \ominus \mathrm{B}^{(\alpha)}$

$$
\begin{aligned}
& =\left(\left[\mathrm{r}_{\mathrm{A}}(\mathrm{i})\right]\left[\mathrm{c}_{\mathrm{A}}(\mathrm{j})\right]\left[\mathrm{a}_{\mathrm{ij}}\right]_{\mathrm{m} \times \mathrm{n}}\right)^{(\alpha)} \ominus\left(\left[\mathrm{r}_{\mathrm{B}}(\mathrm{i})\right]\left[\mathrm{c}_{\mathrm{B}}(\mathrm{j})\right]\left[\mathrm{b}_{\mathrm{ij}}\right]_{\mathrm{m} \times \mathrm{n}}\right)^{(\alpha)} \\
& =\left[\mathrm{r}_{\mathrm{A}}^{(\alpha)}(\mathrm{i}) \ominus \mathrm{r}_{\mathrm{B}}^{(\alpha)}(\mathrm{i})\right]\left[\mathrm{c}_{\mathrm{A}}^{(\alpha)}(\mathrm{i}) \ominus \mathrm{c}_{\mathrm{B}}^{(\alpha)}(\mathrm{i})\right]\left[\mathrm{a}_{\mathrm{ij}}^{(\alpha)} \ominus \mathrm{b}_{\mathrm{ij}}^{(\alpha)}\right]_{\mathrm{m} \times \mathrm{n}} \\
& =[0 \ominus 0,0 \ominus 0][0 \ominus 0,0 \ominus 0][0 \ominus 0,0 \ominus 0] \\
& =[0,0][0,0][0,0]
\end{aligned}
$$

From (2.6.5) and (2.6.6),we get $\mathrm{L}_{\mathrm{ij}}=\mathrm{K}_{\mathrm{ij}}$

In all the cases,

$$
(A \ominus B)^{(\alpha)} \geq A^{(\alpha)} \ominus B^{(\alpha)}
$$

In a similar manner, for the lower cut of IVFMFRCs, we can prove the following theorem.

\section{Theorem 2.7}

If $\mathrm{A}$ and $\mathrm{B}$ are two IVFMFRCs, then

i) $(\mathrm{A} \vee \mathrm{B})_{(\alpha)}=\mathrm{A}_{(\alpha)} \vee \mathrm{B}_{(\alpha)}$

ii) $(\mathrm{A} \oplus \mathrm{B})_{(\alpha)}=\mathrm{A}_{(\alpha)} \oplus \mathrm{B}_{(\alpha)}$

iii) $(\mathrm{A} \ominus \mathrm{B})_{(\alpha)}=\mathrm{A}_{(\alpha)} \ominus \mathrm{B}_{(\alpha)}$ 


\section{Conculsion}

In this paper $\alpha$-cuts of Interval-Valued Fuzzy matrix with Fuzzy Rows and Columns has been introduced. We have also given some definitions based on $\alpha$-cuts with example. Some operator on $\alpha$-cuts are also given. We proved some important theorems of IVFMFRCs using $\alpha$-cuts.

\section{Reference}

[1] A.Kalaichelvi, K.Janofer, $\alpha$-cuts of Triangular fuzzy Numbers and $\alpha$-cuts of Triangular Fuzzy Matrices, Int. Journal of Mathematics Sciences and Applications, Vol 2, No 2, May 2012 635-643.

[2] Madhumangal Pal, Interval-Valued Fuzzy Matrices with Fuzzy Rows and Columns, Fuzzy Information and Engineering (2015): 335-368.f

[3] M.Pal, Fuzzy Matrices with Fuzzy Rows and Columns, Journal of intelligent and Fuzzy Systems to appear.

[4] A.Pal, M.Pal, Some results on Interval-Valued Fuzzy Matrices, The 2010 International conference on E-Business Intelligence Org by Tsinghua University, Kunming, China, Atlantis Press(2010) 554-559,doi;2991/icebi. 2010.39.

[5] A.K.Shyamal, M.Pal, Interval-Valued Fuzzy Matrices, The journal of Fuzzy Mathematics 14(2006) 583-604.

[6] A.K.Shyamal, M.Pal, Two new operators on Fuzzy Matrices, Journal of Applied Mathematics and Computing 15(2004) 91-107.

[7] Amiya K.Shyamal and Madhumangal Pal, Triangular Fuzzy Matrices, Iranian Journal of Fuzzy Systems, Vol 4,No-1(2007) 75-87.

[8] Thomson.M.G, Convergence Of Powers Of a Fuzzy Matrix J. Math. Appl., 57(1997), 476-480. 